\title{
Assessing Injection Techniques in the Treatment of Trigger Finger
}

\author{
John R. Fowler ${ }^{1}$, Lauren Ogrich ${ }^{2}$, Perry Evangelista ${ }^{3}$, Alyssa A. Schaffer ${ }^{1}$ \\ ${ }^{1}$ Temple University Hospital, Department of Orthopaedics, Philadelphia, USA; ${ }^{2}$ Temple University School of Medicine, Philadelphia, \\ USA; ${ }^{3}$ University of Pennyslvania School of Medicine, Philadelphia, USA. \\ Email: john.fowler@tuhs.temple.edu
}

Received June $7^{\text {th }}$, 2012; revised July $8^{\text {th }}$, 2012; accepted August $6^{\text {th }}, 2012$

\begin{abstract}
Background: Trigger finger is characterized by the inability to smoothly flex and extend the digit. Corticosteroids are an accepted non-surgical treatment option and can be delivered via two techniques. While the palmar approach is more commonly used, some have suggested that the mid-axial approach may be less painful for patients and yield higher intrasheath injection rates. The purpose of this study is to compare the accuracy of the palmar and midaxial approaches for delivery of corticosteroids into the flexor tendon sheath using radio-opaque dye in a cadaver model. Methods: A total of 50 injections were performed, 25 via midaxial technique and 25 via palmar technique. A one inch, 25-gauge needle was used to inject $1 \mathrm{~mL}$ of Isovue contrast dye into the flexor tendon sheath under live fluoroscopy. The fluoroscopic images were examined after injection to determine intrasheath versus extrasheath delivery of the dye, with visualization of contrast filling the sheath defining a successful injection. Results: The midaxial approach had a success rate of $52 \%$ compared to the conventional palmar approach success rate of $36 \%, p=0.5$. The ring finger is the most common location of trigger finger and the rates of success were equal between groups for this digit (80\%). Conclusions: Based on our findings, there is no statistical difference in the accuracy of intrasheath injection between the midaxial technique and palmar technique. The midaxial technique can be considered as an alternative to the palmar technique for trigger finger injection.
\end{abstract}

Keywords: Trigger Finger; Injection; Corticosteroids; Palmar and Midaxial Approaches

\section{Introduction}

Trigger finger, also known as stenosing tenosynovitis or stenosing tendovaginitis, is a common condition affecting nearly 100,000 Americans annually [1]. It is characterized by the inability to smoothly flex and extend the digit [1]. The pathophysiology of stenosing tenosynovitis is related to a disproportion between the volume of the flexor sheath and its contents, the flexor tendons [1]. Non-surgical management consists of activity modification, non-steroidal anti-inflammatory medications, and corticosteroid injection [1]. The reported effectiveness of corticosteroid injections has ranged from $25 \%$ - 77\% [26]. A recent systematic review examining the effectiveness of corticosteroid injections found corticosteroid injections to be effective in only $57 \%$ of patients [7]

Although several studies have found extra-sheath corticosteroid injections to have some benefit in the treatment of trigger finger $[3,8]$, the gold standard remains delivery of the corticosteroid into the flexor tendon sheath [1,9]. Two major techniques exist to deliver the corticosteroid into the flexor tendon sheath: the palmar approach and the midaxial approach [1,2,9]. While the palmar approach is more commonly used, some have suggested that the mid-axial approach may be less painful for patients and yield higher intrasheath injection rates $[1,2,9]$. Studies examining the accuracy of intrasheath injection by conventional techniques found delivery into the tendon sheath occurs in only $37 \%-50 \%$ of patients $[8,10]$. If intrasheath delivery of therapeutic medications is an important factor in the treatment of trigger finger, then determining the optimal injection technique is critical.

The purpose of this study is to compare the accuracy of the palmar and midaxial approaches for delivery of corticosteroids into the flexor tendon sheath using radioopaque dye in a cadaver model.

\section{Methods}

The left hands of ten embalmed cadavers were used in this study. The cadaver hands were randomly assigned to receive injection of corticosteroid into the flexor tendon sheath by either the midaxial or palmar approach. For the 
palmar injection technique, the needle was introduced at the level of the A1 pulley and was advanced until resistance was felt. The needle was withdrawn slightly to ensure it was no within the tendon (see Figure 1) and then the contrast was injected. The midaxial technique was performed as described by Carlson [7]. From the radial border of the finger, the needle was inserted into the midlateral area of the proximal phalanx (see Figure 2). The needle was inserted until resistance was felt and then finger was then flexed and extended to confirm that the needle was not in the tendon itself. The contrast dye was then injected.

A one inch, 25-gauge needle was used to inject $1 \mathrm{~mL}$ of Isovue contrast dye into the flexor tendon sheath under live fluoroscopy. Isovue dye was provided by the Department of Radiology. The fluoroscopic images were examined after injection to determine intrasheath versus extrasheath delivery of the dye. A positive outcome was defined as contrast medium tracking proximally and distally through the flexor tendon sheath on fluoroscopic

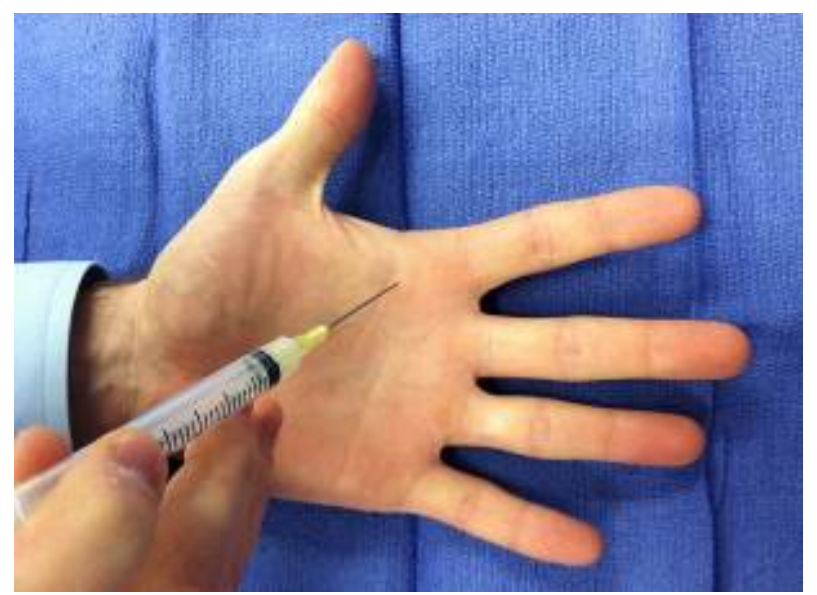

Figure 1. Photograph demonstrating palmar injection technique.

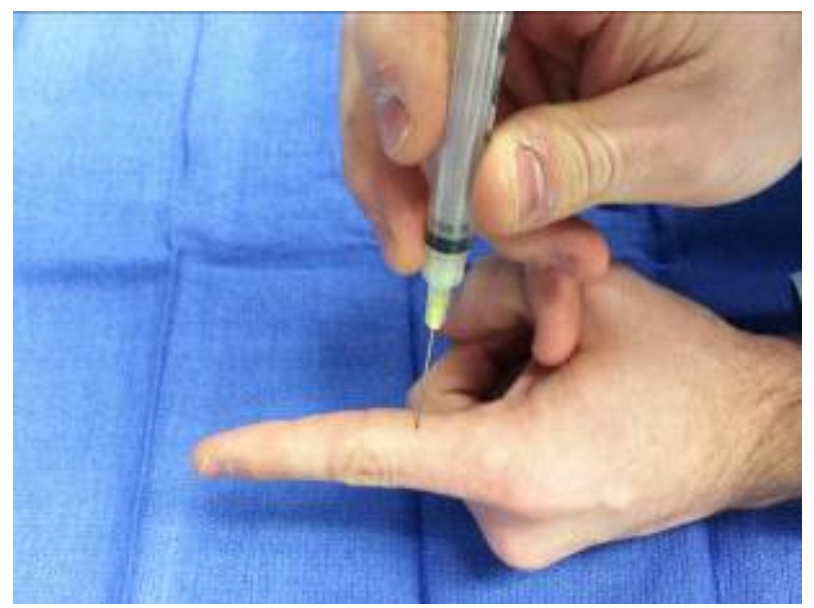

Figure 2. Photograph demonstrating the mid-axial injection technique. images (see Figure 3). A negative outcome was defined as an injection that did not fill the tendon sheath on fluoroscopic images (see Figure 4).

Each finger was injected on each hand for a total of 50 fingers, 25 midaxial and 25 palmar. All injections were performed by a senior orthopaedic resident. The finger, technique, and result were recorded for each hand and images saved for review by the senior author. Statistical analysis was performed using Graphpad (La Jolla, CA) Software. Fisher's exact test was used to determine statistical significance. A power analysis was performed prior to the initiation of the study, which found a sample size of 25 injections would be needed in each group to detect a difference of $30 \%$ between groups, assuming an alpha value of 0.05 and a beta value of 0.8 .

Institutional Review Board exemption was obtained as this was a cadaver study without live subjects.

\section{Results}

The midaxial approach had a success rate of 52\% compared to the conventional palmar approach success rate of $36 \%, p=0.5$. See Table 1 . Injection of the thumb was difficult regardless of the approach, yielding an overall success rate of $0 \%$. If this data is removed, the success rates for the other fingers improves to $65 \%$ for the midaxial and $45 \%$ for the palmar approach, $\mathrm{p}=0.4$. The palmar approach was also found to have a $0 \%$ success rate in the small finger, whereas the midxial approach had a success rate of $80 \%$ in this digit. The ring finger is

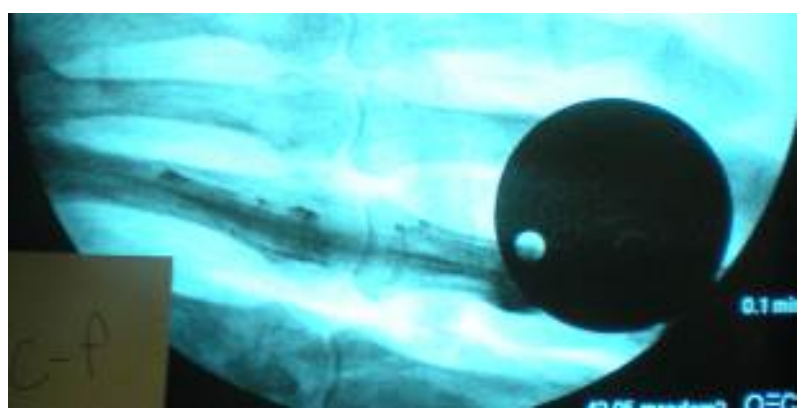

Figure 3. Positive intra-sheath injection, showing contrast tracking throughout the sheath.

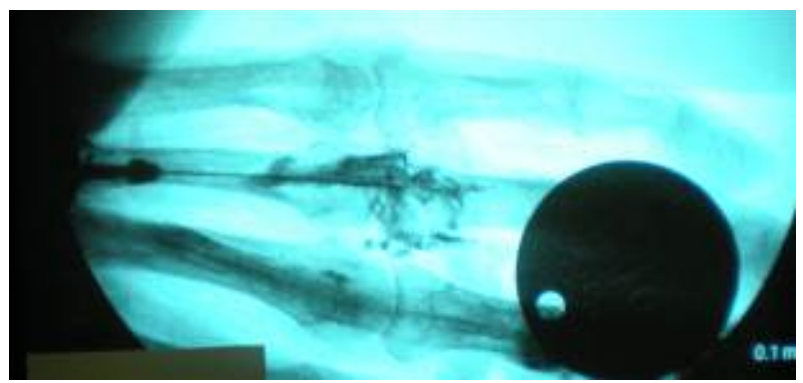

Figure 4. Negative intra-sheath injection, showing contrast tracking outside the sheath. 
Table 1. Injections by location and technique

\begin{tabular}{ccccc}
\hline Finger & $\begin{array}{c}\text { Midaxial } \\
\text { Positive }\end{array}$ & $\begin{array}{c}\text { Midaxial } \\
\text { Negative }\end{array}$ & $\begin{array}{c}\text { Palmar } \\
\text { Positive }\end{array}$ & $\begin{array}{c}\text { Palmar } \\
\text { Negative }\end{array}$ \\
\hline Thumb & 0 & 5 & 0 & 5 \\
Index & 1 & 4 & 2 & 3 \\
Long & 4 & 1 & 3 & 2 \\
Ring & 4 & 1 & 4 & 1 \\
Small & 4 & 1 & 0 & 5 \\
Total & 13 & 12 & 9 & 16 \\
\hline
\end{tabular}

the most common location of trigger finger and the rates of success were equal between groups for this digit (80\%).

\section{Discussion}

Corticosteroids are often the first line of treatment for trigger finger and have relatively high treatment success rates. Unfortunately, intra-tendon sheath injections given via a palmar approach injection technique are painful for the patient and can cause tendon rupture if the injection releases corticosteroid in the tendon itself. The midaxial technique has similar success rates at treating the symptoms of the triggering digit as the palmar approach injection technique and has been reported to be less painful $[1,3,7]$. The current study specifically addressed the injection technique and the ability to deliver contrast medium into the flexor tendon sheath.

This study has several limitations. The cadavers used in this study were embalmed and this could affect the distensibility of the flexor sheath and possibly make intrasheath injection more difficult. Due to the limited availability of cadaver hands, the number of injections to each finger ( 5 in each group) is relatively low. This study is underpowered, as an a priori power analysis found that 25 injections would be needed to show a difference of $30 \%$ in each group. The difference found in this study, however, was only $19 \%$, which would have required over 150 injections. It would be interesting to inject a larger number of fingers to determine if the techniques were more similar in success rates with a larger sample size. A single surgeon performed all injections, introducing operator bias. The determination of a positive versus negative injection is also subject to bias, although the images were reviewed by a senior hand surgeon who was not present for the injections and the agreement was $100 \%$.

To our knowledge, this is the first study to use contrast dye in a cadaver model to determine the intrasheath accuracy of the palmar and midaxial injection techniques. Although underpowered, our findings demonstrate no difference in the accuracy of intrasheath injection be- tween the midaxial technique (52\%) and palmar technique (36\%), $\mathrm{p}=0.5$. Our data is comparable to previously published studies that demonstrated intrasheath injection rates of $37 \%$ - 57\% [10-12]. We were unable to deliver the contrast medium into the thumb flexor tendon sheath with either approach, which also coincides with earlier studies $[8,10]$. It is possible that the lack of successful treatment of trigger finger with corticosteroid injections is due to the inability to deliver the steroids into the sheath.

\section{Conclusion}

Based on our findings, there is no statistical difference in the accuracy of intrasheath injection between the midaxial technique and palmar technique. The midaxial technique can be considered as an alternative to the palmar technique for trigger finger injection.

\section{REFERENCES}

[1] M. J. Saldana, "Trigger Digits: Diagnosis and Treatment," Journal of the American Academy of Orthopaedic Surgeons, Vol. 9, No. 4, 2001, pp. 246-252.

[2] N. Buch-Jaeger, G. Foucher, S. Ehrler and D. Sammut, "The Results of Conservative Management of Trigger Finger: A Series of 169 Patients," Annals of Hand \& Upper Limb Surgery, Vol. 11, No. 3, 1992, pp. 189-193.

[3] K. Kazuki, T. Egi, M. Okada and K. Takaoka, "Clinical Outcome of Extrasynovial Steroid Injection for Trigger Finger," Hand Surgery, Vol. 11, No. 1-2, 2006, pp. 1-4. doi:10.1142/S0218810406003115

[4] M. Lambert, R. Morton and J. Sloan, “Controlled Study of the Use of Local Steroid Injection in the Treatment of Trigger Finger and Thumb," The Journal of Hand Surgery: Journal of the British Society for Surgery of the Hand, Vol. 17, No. 1, 1992, pp. 69-70. doi:10.1016/0266-7681(92)90014-S

[5] M. R. Marks and S. F. Gunther, "Efficacy of Cortisone Injection in Treatment of Trigger Fingers and Thumbs," Journal of Hand Surgery, Vol. 14, No. 4, 1989, pp. 722-727.

[6] D. Murphy, J. M. Failla and M. P. Koniuch, "Steroid versus Placebo Injection for Trigger Finger," Journal of Hand Surgery, Vol. 20, No. 4, 1995, pp. 628-631.

[7] C. Carlson Jr. and R. Curtis, "Steroid Injection for Flexor Tenosynovitis,” Journal of Hand Surgery, Vol. 12, No. 2, 1984, pp. 286-287.

[8] J. S. Taras, J. S. Raphael, W. T. Pan, F. Movagharnia and D. G. Sotereanos, "Corticosteroid Injections for Trigger Digits: Is Intrasheath Injection Necessary?” Journal of Hand Surgery, Vol. 23, No. 4, 1998, pp. 717-722.

[9] A. Freiberg, R. Mulholland and R. Levine, "Nonoperative Treatment of Trigger Fingers and Thumbs," Journal of Hand Surgery, Vol. 14, No. 3, 1989, pp. 553-558.

[10] M. Kamhin, J. Engel and M. Heim, "The Fate of Injected Trigger Fingers,” Hand, Vol. 15, No. 2, 1983, pp. 218- 
220. doi:10.1016/S0072-968X(83)80018-7

[11] S. B. Fleisch, K. P. Spindler and D. H. Lee, “Corticosteroid Injections in the Treatment of Trigger Finger: A Level I and II Systematic Review," Journal of the American Academy of Orthopaedic Surgeons, Vol. 15, No. 3, 2007, pp. 166-171.
[12] D. Ring, S. Lozano-Calderón, R. Shin, P. Bastian, C. Mudgal and J. Jupiter, “A Prospective Randomized Controlled Trial of Injection of Dexamethasone versus Triamcinolone for Idiopathic Trigger Finger," Journal of Hand Surgery, Vol. 33, No. 4, 2008, pp. 516-522. 